\title{
Histochemical and Molecular Characterization of Spongiosal Cells in Native Tissue, Two- and Three-Dimensional Cultures of Rat Aortic Valve
}

\author{
Vibudha Nanduri, ${ }^{1}$ Beheita Moein, ${ }^{1}$ Avinash Raj Thatipalli, ${ }^{1}$ \\ Gopal Pande, ${ }^{1}$ and Anasuya Ganguly ${ }^{2}$ \\ ${ }^{1}$ Council of Scientific and Industrial Research-Centre for Cellular and Molecular Biology, Hyderabad 500007, India \\ ${ }^{2}$ Birla Institute of Technology and Sciences, K. K. Birla Campus, Zuarinagar, Goa 403726, India
}

Correspondence should be addressed to Gopal Pande; pandeji10@gmail.com and Anasuya Ganguly; ganguly@goa.bits-pilani.ac.in

Received 7 September 2015; Revised 2 December 2015; Accepted 15 December 2015

Academic Editor: Victor H. Casco

Copyright @ 2016 Vibudha Nanduri et al. This is an open access article distributed under the Creative Commons Attribution License, which permits unrestricted use, distribution, and reproduction in any medium, provided the original work is properly cited.

\begin{abstract}
The histocytochemical and molecular analysis of cells that constitute the aortic valve (AV) of the rat heart was done in this study. We have focussed on the identity of cells in the spongiosal layer of the valve by immunofluorescence studies using lineage specific markers and cytochemical staining. We have established two-dimensional (2D) cultures of cells from isolated rat AV leaflets and maintained endothelial and interstitial valvular cells (IVC) over a period of six to eight weeks. Using "passage 0" cells from 2D valvular cultures, we could reconstruct the three-dimensional (3D) valvular tissue in collagen gels that showed very similar cellular organization and marker expression profile, as that of the native tissue. Lineage specific markers in the native tissue and cell cultures were studied by Reverse Transcriptase-PCR and immunofluorescence for VCAM-I, $\alpha$-SMA, collagen I, CD71, collagen II, and Ecadherin markers. This is the first report on the identification of cell lineages in the spongiosal layer of AV and the successful reconstruction of $3 \mathrm{D}$ valvular tissue from primary cell cultures of $\mathrm{AV}$.
\end{abstract}

\section{Introduction}

Human and murine hearts comprise four valves (aortic, pulmonary, mitral, and tricuspid valve) that are located on a single valvular plate (Figure 1(a)(i)). Our interest was to study the cellular architecture of aortic valve (AV) in rat hearts which is located between the left and right atrioventricular annuli and connects the left ventricle and the ascending aorta [1]. AV is one of the semilunar valves and it comprises three leaflets attached to a common annular ring; each leaflet is composed of three different layers, namely, the fibrosa $(\mathrm{F})$, spongiosa (S), and ventricularis (V) (Figure 1(a)(iv)). The F layer is situated away from the blood flow and constituted by dense collagen fibres; $\mathrm{V}$ layer, located adjacent to the blood flow, is elastic in nature and it contains thin collagen fibres; $\mathrm{S}$ layer is situated between the $\mathrm{F}$ and $\mathrm{V}$ layer and it has high proteoglycan and elastin content that facilitates the valve to absorb high pressure and allow flexible movement. Each layer in the valve leaflet exhibits their specific functions due to the composition and organization of the cells in the extracellular matrix. Considering the F layer, it provides immense strength to the valve during blood flow. The $\mathrm{S}$ layer enhances the movement between the layers and acts as a shock absorber during closing of the valves. Due to the elastic nature of $\mathrm{V}$ layer, the extracellular matrix on the surface of the valve leaflet is stretched and retracted during the cardiac cycle. The mechanical properties of the three layers of leaflets provided by the collagen and elastin fibres help in proper function of heart during the cardiac cycle [2-5]. So far, the cellular composition of $S$ layer has not been studied in detail and this layer is considered to have very few cells, the identity of which is not clear.

Due to various disorders and malfunctions associated with heart valves, ex vivo reconstruction of valves, using tissue engineering approaches, has been attempted in many labs [6-13]. However, as most of these methods have used 


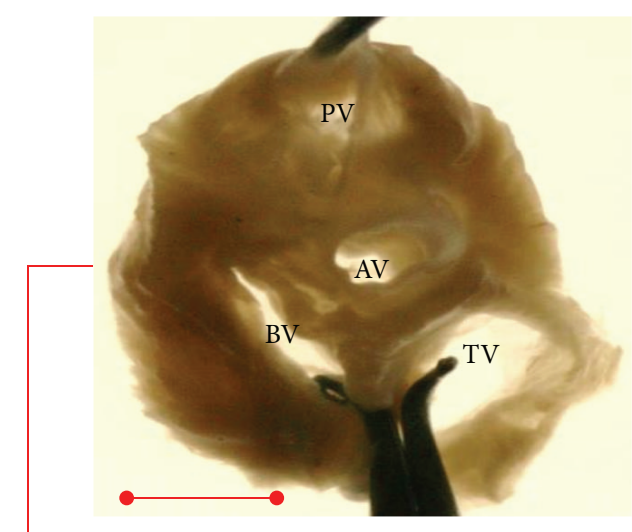

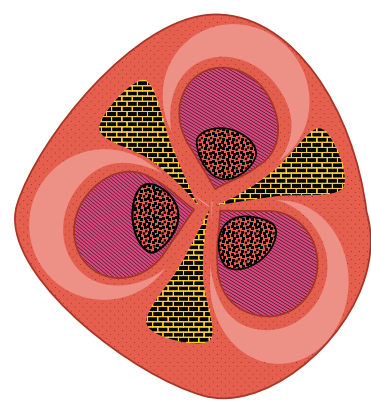

Aortic wall 的 Lamina spongiosa Annulus

Leaflets (i)

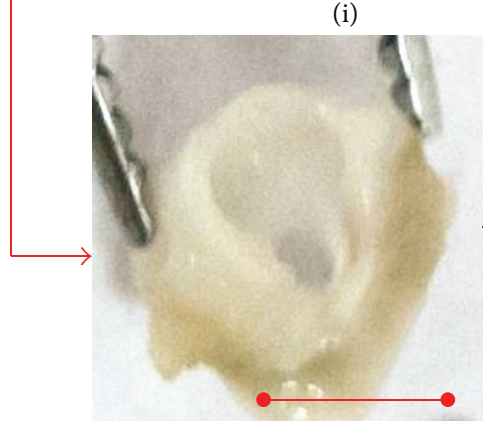

(iii)

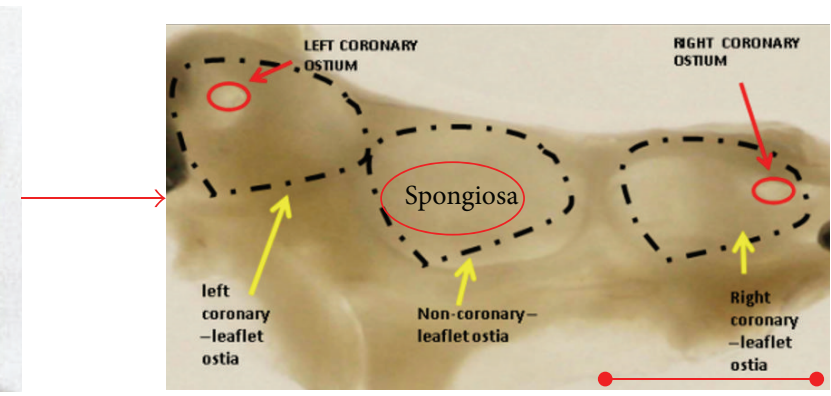

(iv)

(a)

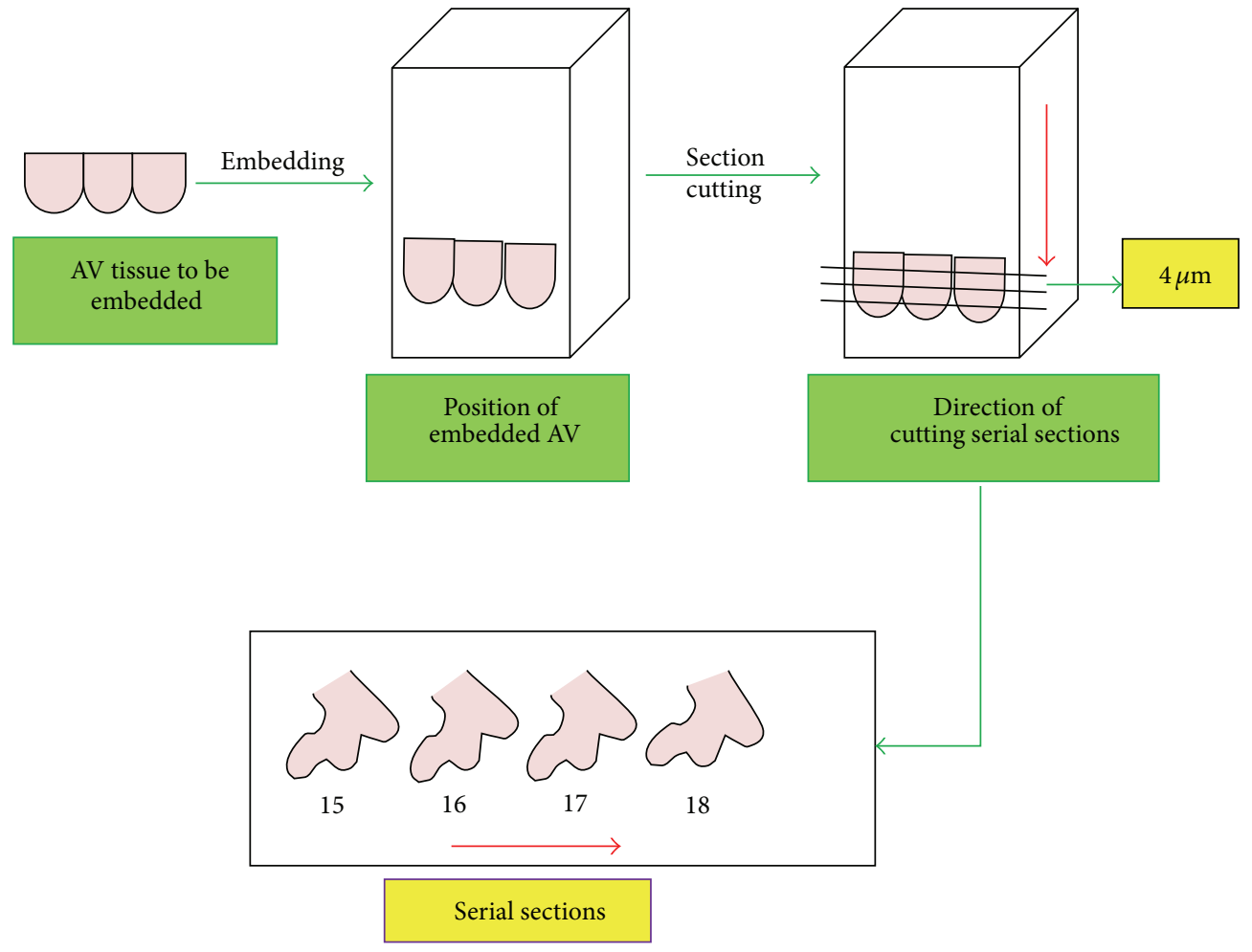

(b)

FIgURE 1: (a) (i) Layout of all valves in the rat valvular plate: AV: aortic valve, PV: pulmonary valve, BV: bicuspid valve, TV: tricuspid valve (scale bar represents $3 \mathrm{~mm}$ ); (ii) schematic representation of the anatomy of AV; (iii) arrangement of leaflets in the AV in its closed form (scale bar represents $2 \mathrm{~mm}$ ); and (iv) arrangement of leaflets in the AV in its open form (scale bar represents $2 \mathrm{~mm}$ ). (b) Orientation of AV leaflets for serial sectioning. 
nonbiological or acellular materials, the identity of cells in the reconstructed valves remains unclear.

Here, we have attempted to identify cells in the S layer of rat AV by studying lineage specific marker expression and doing cytochemical analysis of the primary tissue and cultured cells. The specific location and orientation of rat AV leaflets and isolation of primary tissue from the $S$ layer have been illustrated in Figures 1(a) and 1(b). We have adopted tissue engineering methodologies for establishing $2 \mathrm{D}$ and $3 \mathrm{D}$ cultures of rat $\mathrm{AV}$ cells and characterized the cultured cell types by immunocytochemistry and molecular analysis. These $3 \mathrm{D}$ cultures allow the cells to grow with their neighboring conditions in all three dimensions. These cultures are mainly used for in vivo cells and models. 3D cultures help in cell-cell or cell-matrix interactions at higher rates, especially during the presence of a biological material, like that of collagen.

\section{Materials and Methods}

2.1. Collection of Wistar Rat Aortic Valve. All studies were performed on the hearts of healthy Wistar rats (8-12 weeks old) after fulfilling the requirements of Institutional Animal Ethics Committee (registration number: CPCSEA 20/1999). The area around the junction of the aorta with the right ventricle was dissected and the entire valvular plate containing the four heart valves was isolated. The AV was separated and cut open to expose the leaflets (see Figures 1(a)(i), 1(a)(iii), and $1(\mathrm{a})(\mathrm{iv})$ ). Figure $1(\mathrm{a})(\mathrm{ii})$ describes the layout of the $S$ layer in the leaflet. The valvular tissue was made free from all the surrounding muscle and the three valvular leaflets, with an average wet weight $0.2 \mathrm{~g}$, were isolated and processed for further studies.

\subsection{Tissue Fixation and Embedding}

2.2.1. Histocytochemical Methods. The AV tissue (measuring about $0.25 \mathrm{~cm}^{2}$ ) is longitudinally opened, fixed in $10 \%$ buffered formalin for 7 days, processed sequentially in grades of $70 \%, 80 \%, 95 \%$, and $100 \%$ isopropyl alcohol and chloroform (each step of processing with different grades of alcohol was done for 25 minutes), and finally embedded into wax blocks (Figure 1(b)). The procedure was followed as per the protocol described elsewhere [14]. $4 \mu \mathrm{m}$ thick serial sections of the tissue (Figure 1(b)) were cut using a Leica RM 2135 microtome and stained using Hematoxylin and Eosin ( $\mathrm{H}$ and $\mathrm{E})$, Toluidine Blue (TB) for total proteoglycan [14], and Masson's trichrome (MT) for total collagen (procedure number HT15, Sigma-Aldrich, India).

2.2.2. Tissue Processing. Primary valvular cells from AV were prepared according to a standardized protocol described in literature elsewhere $[15,16]$. Description of experiments and methodology of both 2D and 3D cultures are given below.

Two-Dimensional (2D) Cultures. Four normal AV leaflets, devoid of any extra muscular tissue and the aortic wall, were separated from the valvular plate and pooled. The pooled leaflets were minced in small volumes $(200-300 \mu \mathrm{L})$ of DMEM (DMEM high glucose, Sigma-Aldrich, India) and the cell/tissue suspension was filtered through $100 \mu \mathrm{m}$ sterile nylon mesh (BD Falcon, India). The tissue pieces that were left on top of the mesh were sequentially treated in enzymatic solutions, prepared in 1x PBS, and filter sterilized using $0.22 \mu \mathrm{m}$ syringe filters in the following order: $0.2 \%$ trypsin (Cat. number T4049, Sigma-Aldrich, India) and $0.08 \%$ collagenase type IV (Cat. number C5138, Sigma-Aldrich, India), at $37^{\circ} \mathrm{C}$ for 30 minutes each. Cells obtained after enzymatic treatment were collected by centrifugation, counted and plated onto T25 culture flask (BD Falcon, India) at an average seeding density of $4 \times 10^{5}$ cells $/ \mathrm{cm}^{2}$, and maintained in complete medium (CM) (DMEM comprising 1x glutamax and $1 x$ penicillin-streptomycin (both from Gibco, India), 10\% FBS (Sigma-Aldrich, India)) for a period of 20 days, with alternate day media changes. On reaching confluence, the cells were subcultured till passage 3 (P3) and maintained for 7 days in each passage. At each passage, cells were both cryopreserved, processed for immunocytochemical and molecular analysis and to establish 3D cultures.

Three-Dimensional Cultures (3D). Valvular cells from AV grown in $2 \mathrm{D}$ cultures for 20 days (P0) were suspended in collagen type I gels (Cat. number 3447-020-01, Invitrogen, India), prepared at $2 \mathrm{mg} / \mathrm{mL}$ in alkaline $\mathrm{PBS}$ at the concentration of $1.5 \times 10^{4}$ cells $/ \mathrm{mL} ; 100 \mu \mathrm{L}$ of this suspension was added to Millicell 24-well culture inserts (Cat. number PIHT 12R 48, India) placed in a 24-well culture plate (BD Falcon, India), under aseptic conditions. Same volume of plain collagen gel devoid of valvular cells was used as a control. Both the gels were left undisturbed for 60 minutes at $37^{\circ} \mathrm{C}$, after which the gel gets solidified. Once the gel gets firm, $200 \mu \mathrm{L}$ of CM was added in each well, and the cultures were maintained for 30 days with media changes twice a week. After the 30-day period, the collagen gels with and without the valvular cells were processed for histology, immunofluorescence, and RTPCR.

2.2.3. Immunofluorescence. Various antibodies were used to study the different cell lineage patterns that were expressed in the native $\mathrm{AV}, 2 \mathrm{D}$ valvular cells, and 3D-reconstructed $\mathrm{AV}$. Primary antibodies (all obtained from Abcam, USA) used for the study were as follows: rat anti-VCAM1 (ab78712), rabbit anti- $\alpha$ SMA (ab5694), rabbit anti-collagen I (ab34710), rabbit anti-collagen II (ab53047), rabbit anti-E cadherin antibody (ab53033), and mouse anti-CD71 (ab112215). All the secondary antibodies (obtained from Invitrogen, India), conjugated with Alexa Fluor 594 used for the study, included goat anti-rabbit IgG (A11012), goat anti-rat IgG (A11007), and goat anti-mouse IgG (A11032). The same immunofluorescence protocol was used for sections of native and 3D-reconstructed aortic valves. $4 \mu \mathrm{m}$ thick sections were deparaffinised with xylene, treated with different ethanol concentrations in sequence, and placed in sodium citrate buffer $(\mathrm{pH}, 6.0)$ for heat-mediated antigen retrieval. For $2 \mathrm{D}$ cultures of valvular cells, the cells were first grown on $18 \mathrm{~mm}^{2}$ coverslips and then fixed with $4 \%$ paraformaldehyde and dried. For sections as well as the cells, blocking of nonspecific 

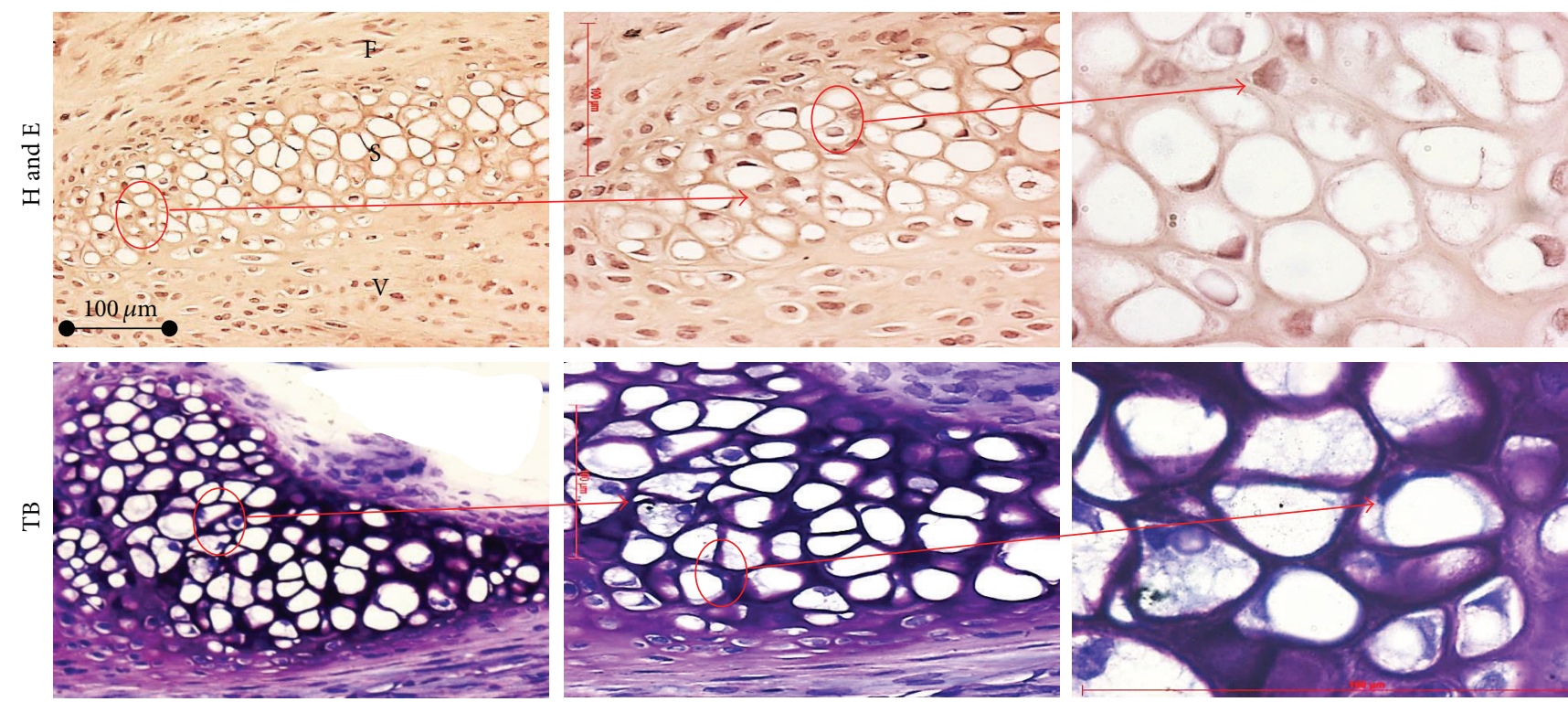

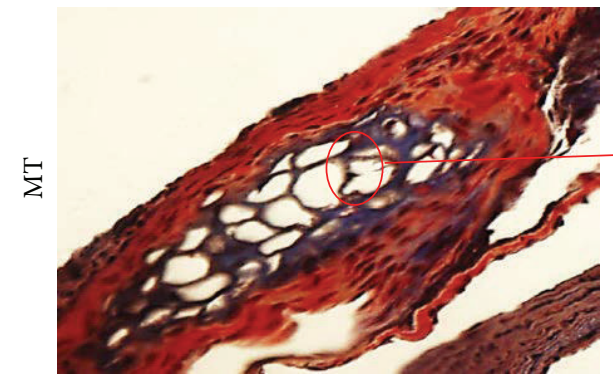

(a)
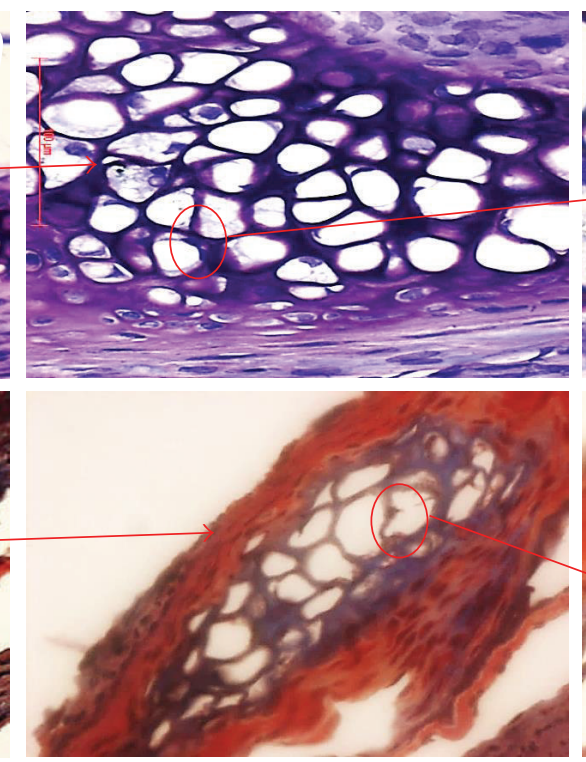

(b)
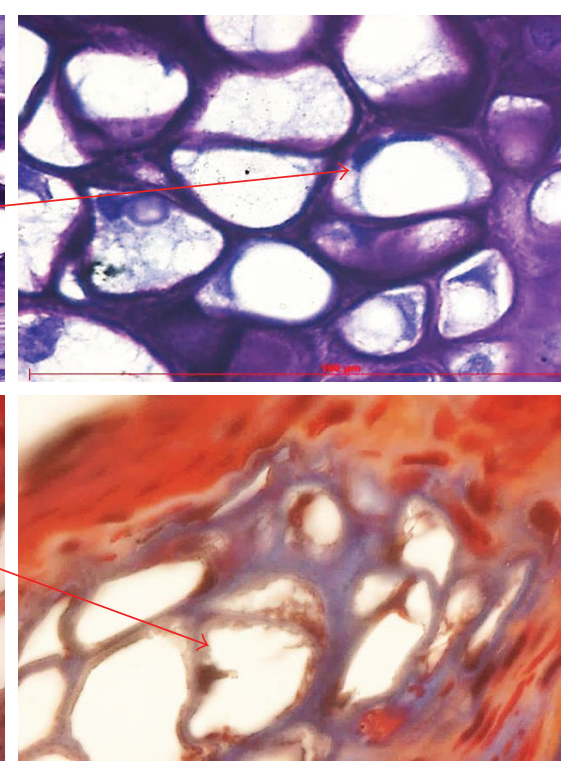

(c)

FIGURE 2: Histological staining of the S layer in the native AV leaflet with Hematoxylin and Eosin ( $\mathrm{H}$ and E), Toluidine Blue (TB) and Masson's trichrome (MT) at 20x (a), 40x (b), and 100x (c) magnification. Arrows indicate location of cells. Scale bar is $100 \mu \mathrm{m}$.

antibodies was done by treatment with $3 \%$ bovine serum albumin (BSA) in PBS. The sections and the cells were treated with $1: 100$ dilutions of their respective primary antibodies for 1 hour at room temperature and washed with PBS, and then 1:1000 dilutions of their relevant secondary antibodies were added and mounted with DAPI fluoroshield. Specific fluorescence for Alexa Fluor 594 goat anti-rabbit IgG antibody and DAPI was observed in a Zeiss Apotome fluorescence microscope comprising a 20x Plan-Apochromatic objective and filter settings of $594 \mathrm{~nm} / 620 \mathrm{~nm}$ and $350 \mathrm{~nm} / 470 \mathrm{~nm}$ as the excitation/emission wavelengths. Image analysis studies were done using AxioVision Rel 4.7 software (Zeiss).

2.2.4. Gene Expression Profiling. Total RNA was extracted from four pooled native AVs $(1.2 \mathrm{mg} / \mathrm{mL})$, valvular cells$2 \mathrm{D}$ cultures $(0.78 \mathrm{mg} / \mathrm{mL})$ and $3 \mathrm{D}$-reconstructed AV tissue $(1 \mathrm{mg} / \mathrm{mL}$ ) using TRIzol reagent (Cat. number 1015, Bioserve, India). The extracted RNA was purified by DNAse (Cat. number 18068-015, Invitrogen) treatment, and $1 \mu \mathrm{g}$ RNA of each sample was reverse transcribed using Super-Script III First-Strand Synthesis System for RT-PCR (Cat. number 18080-051, Invitrogen, India). The PCR was performed using the DNA Engine Peltier Thermal Cycler (Bio-Rad, India) for analysing the expression of high performance liquid chromatography- (HPLC-) purified nucleotide primers of rat GAPDH (the house-keeping gene) [17], collagen II [18], collagen I, $\alpha$ SMA [19], VCAM I [20], E-cadherin [21], and CD71 [22] genes, obtained from Bioserve, India. Primer sequences for these genes are taken from the references mentioned above. To analyse GAPDH expression, the following PCR conditions were considered: hot start, $95^{\circ} \mathrm{C}, 2$ minutes; 30 cycles of denaturation, $95^{\circ} \mathrm{C}, 30$ seconds; annealing, $57.5^{\circ} \mathrm{C}, 30$ seconds; and elongation, $72^{\circ} \mathrm{C}, 30$ seconds. This was followed by final extension at $72^{\circ} \mathrm{C}, 5$ minutes. The PCR conditions to study the expression of the remaining genes were similar to those used for GAPDH, but with the changes in the annealing temperatures of $58^{\circ} \mathrm{C}$ for collagen II, $65^{\circ} \mathrm{C}$ for collagen I and $\alpha \mathrm{SMA}, 58^{\circ} \mathrm{C}$ for VCAM I, $55^{\circ} \mathrm{C}$ for E-cadherin, and $60^{\circ} \mathrm{C}$ for CD71. Safe View Classic (Cat. number G108, Applied Biological Materials, India) in $1 \%$ agarose gel was used to visualize the amplified products.

\section{Results}

3.1. Native $A V$ Histology. Figure 2 illustrates the $\mathrm{H}$ and $\mathrm{E}$ staining which was used to delineate the cellular organization of the three layers of AV. F layer showed low cell content, $\mathrm{S}$ layer had large nuclei and rich matrix, and the $\mathrm{V}$ 

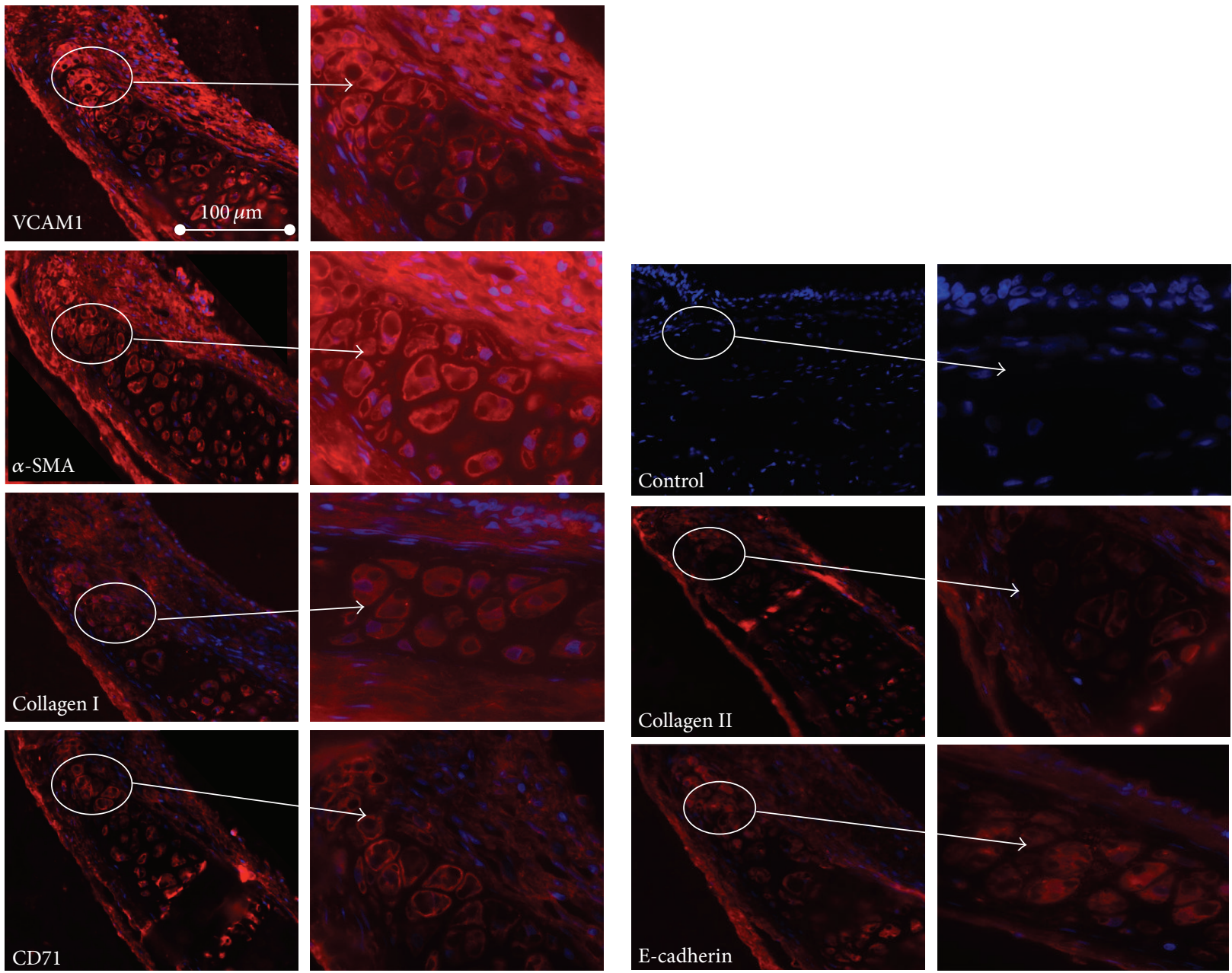

(a)

(b)

FIGURE 3: Immunofluorescence of native AV leaflets showing expression of markers with high expression (a) and low expression (b) at 20x magnification (exhibiting all 3 layers) and corresponding 63x magnified images (showing only the S layer). Scale bar is $100 \mu \mathrm{m}$.

layer exhibited higher cell number with each cell showing high cytoplasmic content. TB staining revealed a differential proteoglycan content in all the three layers of which $S$ layer showed the highest expression. MT stained tissue highlighted the total collagen content in all the layers and once again the $\mathrm{S}$ layer showed the highest collagen specific staining.

3.2. Lineage Specific Marker Expression. Marker expression to identify specific cell lineages was studied by immunofluorescence as described. Figure 3 shows that VCAM-I (endothelial cells) and $\alpha$ SMA (interstitial valvular cells) were expressed in all three layers with maximum expression in the S layer; CD71 (mesenchymal cells) and collagen I (fibroblastic cells) were expressed at higher levels in the V layer; E-cadherin (epithelial cells) and collagen II (chondrocytic cells) were expressed at very low levels in all the three layers. These observations confirm that VCAM I and $\alpha$ SMA are the main markers expressed in the $\mathrm{S}$ layer indicating that this layer is composed mainly of IVC and endothelial cells.
3.3. Two-Dimensional Culture Studies. Primary cells isolated from AV leaflets as described were maintained in $\mathrm{CM}$ for a period of 20 days (P0) or 7 days (P1, P2, and P3 each). Figure 4 describes the phase contrast microscopy images of the cells maintained from P0 to P3. We can clearly observe that there is a gradual decrease in cell size from P0 to P3, and this could be due to the cellular and matrix differentiation that occur during the culture process.

To confirm cell lineages in the 2D cultures, immunofluorescence of VCAM I, $\alpha$ SMA, collagen I, collagen II, Ecadherin, and CD71 was performed. This analysis was done in 10 different experiments that showed similar results as shown in Figure 5. Though the cell size decreased gradually during P0-P3 (Figure 4), the expression of VCAM I and $\alpha$ SMA remained consistently high through the passages. Similar to the observations in primary AV tissue (Figure 3), expression of collagen I and CD71 showed moderate expression and expression of collagen II and E-cadherin was at very low levels. These data confirm that, in 2D cultures, maintenance of endothelial cells and IVC was more than other cell types. 

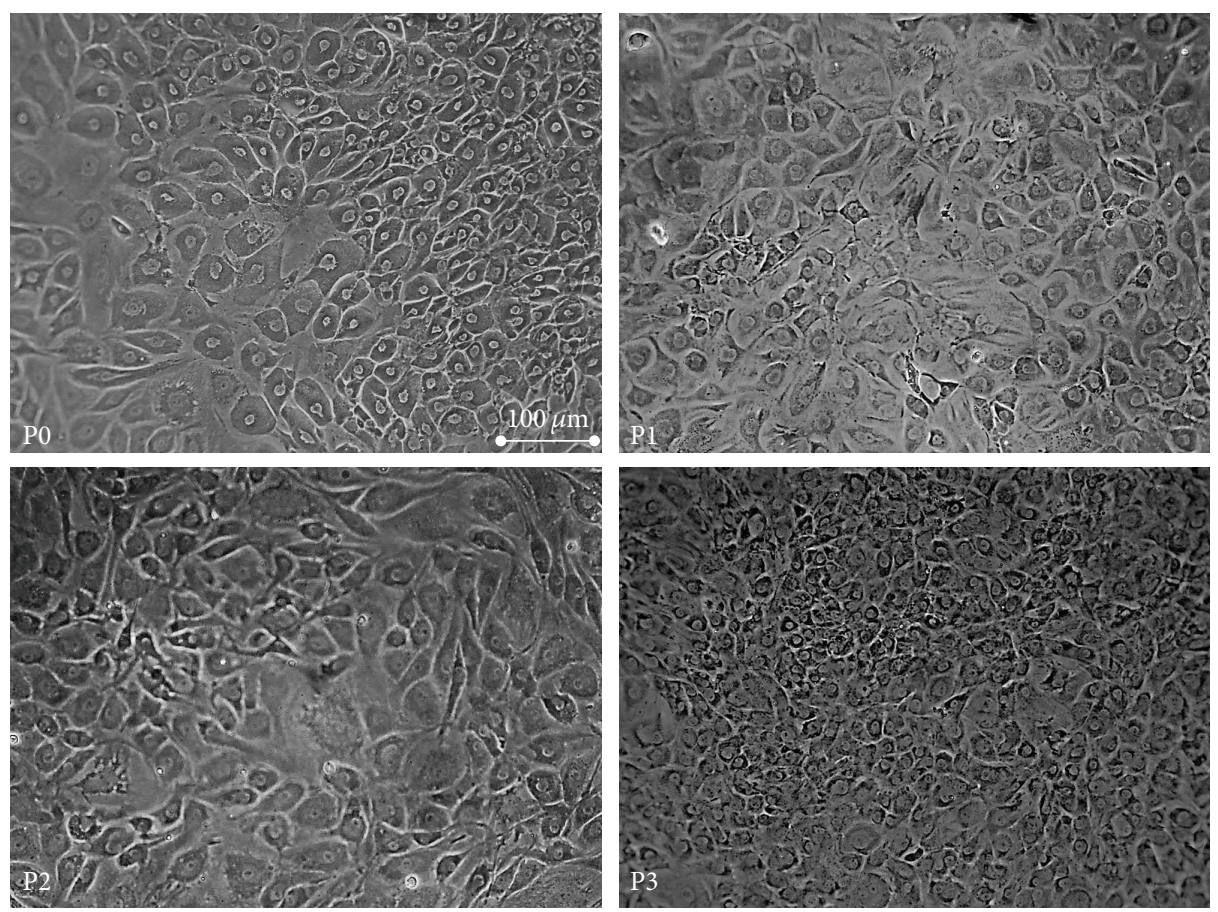

FIGURE 4: Phase contrast images of cells grown in 2D cultures of AV leaflets at 10x magnification. The cultures were maintained from P0 to P3; change in cell size with increase in passage number is visible. Scale bar is $100 \mu \mathrm{m}$.
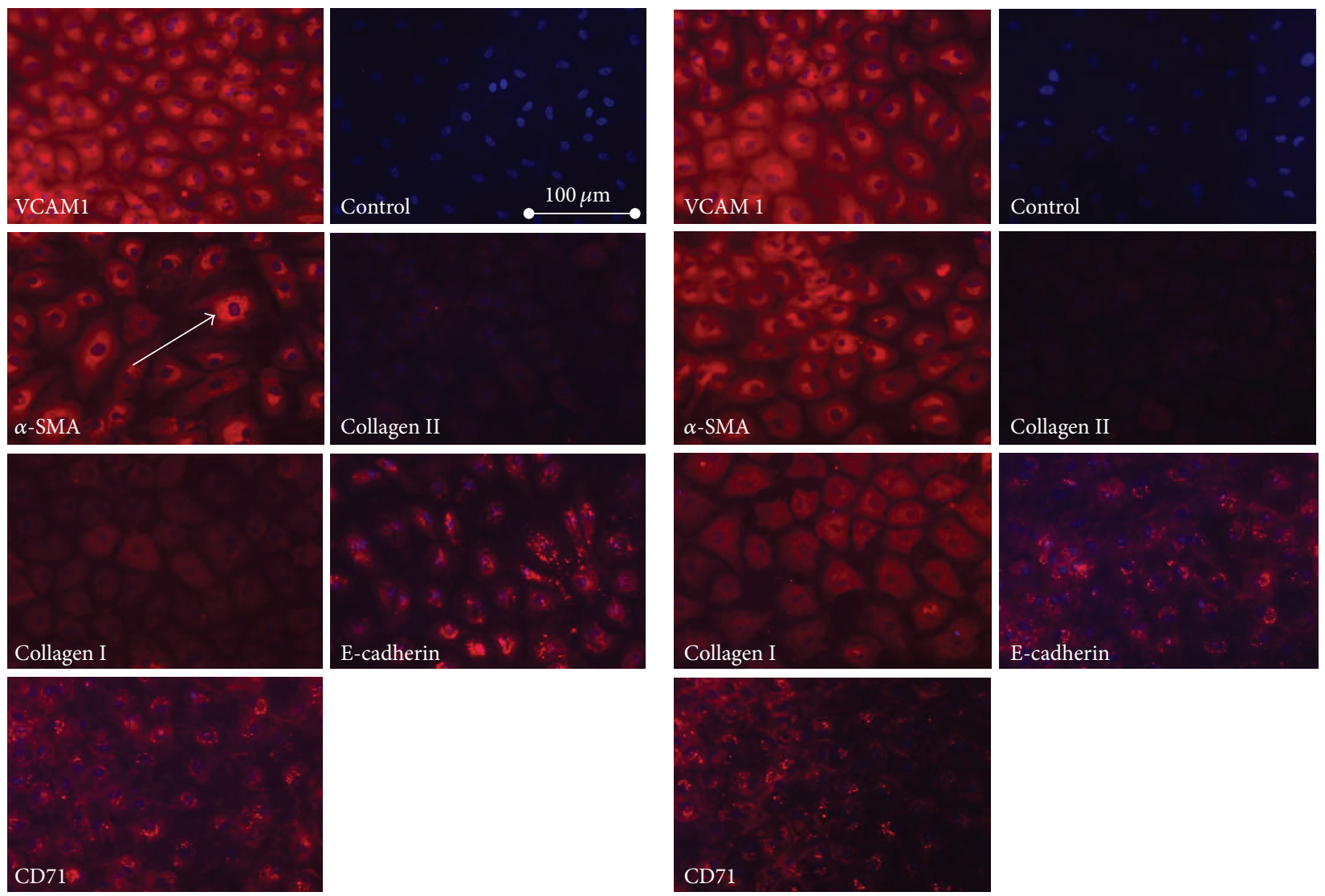

(a)

(b)

Figure 5: Immunofluorescence of cells in AV leaflet 2D cultures at P0 (a) and P3 (b), at 20x magnification. Scale bar is $100 \mu \mathrm{m}$. 


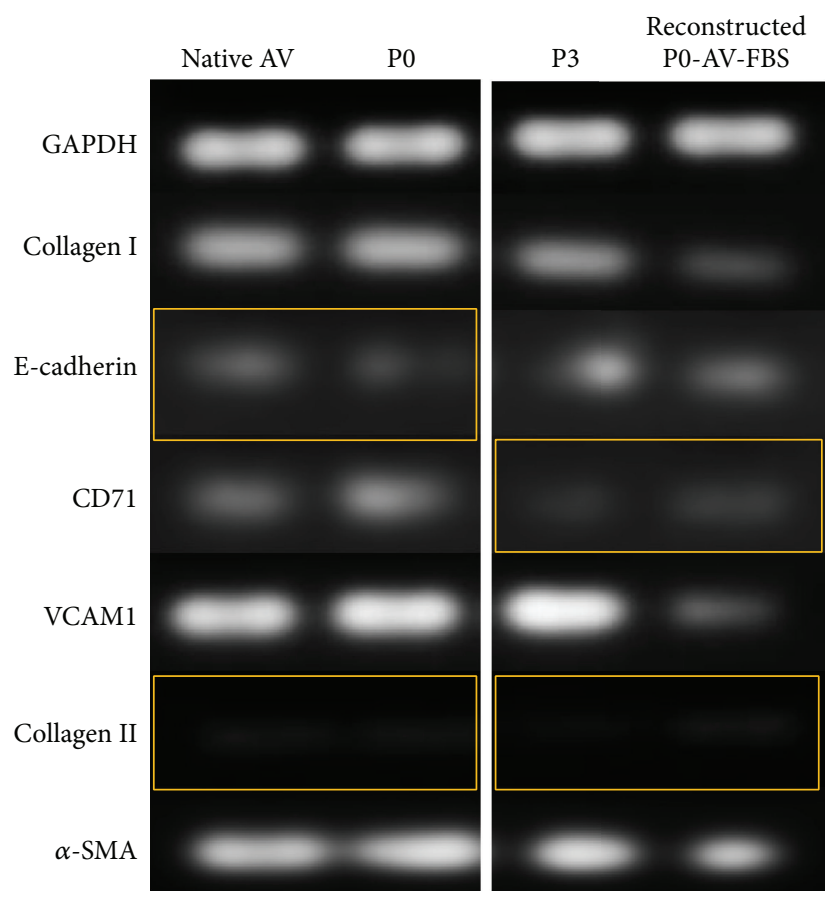

FIGURE 6: RT-PCR products for the respective markers expressed in native, 2D, and 3D cultures of AV leaflets.

3.4. Three-Dimensional Culture Studies. Primary AV leaflet cells first grown in 2D cultures were collected 3 weeks after plating and then transferred into collagen type I gels in 24well millicell culture inserts as described in Section 2. Collagen gels, with and without AV cells, were stained with $\mathrm{H}$ and E, TB, and MT and the results are shown in Supplementary Figure 1A in Supplementary Material available online at http://dx.doi.org/10.1155/2016/7680701. Interestingly, $\mathrm{H}$ and E staining pattern of cells in the $3 \mathrm{D}$ culture showed a systematic arrangement of the F, S, and L layers. TB and MT staining of the gels also showed maximum expression of total proteoglycan and collagen contents in S layer. These staining patterns of $3 \mathrm{D}$ gels mimic the distribution cell in the native AV tissue.

Immunofluorescence for VCAM I, $\alpha$ SMA, collagen I, CD71, collagen II, and E-cadherin in 3D-reconstructed AV is shown in Supplementary Figure 1B. CM grown AV tissue clearly depicts the three layers, with higher expression of VCAM I and $\alpha$ SMA proving higher cellular content for endothelial and valvular interstitial cells, respectively. Collagen I and CD71 are expressed moderately all through the tissue, and collagen II and E-cadherin are expressed at very low levels.

3.5. Gene Profiling Analysis. A comparative study of native AV cells acquired from 2D and 3D AV culture systems was done by RT-PCR. Equal quantities of total RNA was used to study GAPDH (control), VCAM I, $\alpha$ SMA, collagen II, collagen I, CD71, and E-cadherin expression levels. Fifteen independent experiments were done and similar results that are depicted in Figure 6 were confirmed. High levels of expression of VCAM I and $\alpha$ SMA genetic markers were observed in native $\mathrm{AV}, 2 \mathrm{D}$, and $3 \mathrm{D}$ cultures. Collagen II and E-cadherin remained consistently low in all of the three sources. Collagen I and CD71 showed moderate expression from the sources selected.

Our results confirm that among other cellular lineages, the endothelial and IVC origin cells were main representatives of the valvular tissue in $3 \mathrm{D}$ cultures and primary $\mathrm{AV}$ tissue.

\section{Discussion}

Several earlier reports have described the histochemical architecture of heart valves especially the aortic valve $[1,23-$ 25]. These studies have shown that all valves have a layered organization of which some layers face the direction of blood flow and some are away from it. The composition of the cells in these layers is related to the functions of the concerned layer $[1-4,23]$. In the AV, three clear cut layers have been observed of which the ventricularis and the fibrosa that compose the external surfaces of the valve are better studied $[2,26]$. The spongiosal layer which lies in the middle of the valve plays a crucial role in determining the flexibility and elasticity of the valve. As several diseases of AV affect its elastic and mechanical properties, therefore it is necessary to know the cellular and molecular composition of the $S$ layer in better detail.

In this report, we have found that the $S$ layer is primarily composed of endothelial and interstitial cells although their number is very low. As per the gross anatomy (Figure 2), the cells show a large nuclear organization and are deeply embedded within fenestrated structures that are distributed all through the S layer. Decrease in the cell number in $2 \mathrm{D}$ cultures is noticed which might be due to the rearrangement of cellular and matrix properties, the main feature of spongiosa 
layer. The cells express high levels of VCAM and $\alpha$ SMA and low or nil other markers. These properties of the spongiosal cells could also be reproduced in the $2 \mathrm{D}$ cultures at early passages and in the reconstructed tissues in the $3 \mathrm{D}$ cultures.

Based on these observations, we propose that future studies on the $\mathrm{AV}$ valve regeneration or reconstruction ex vivo can be achieved if correct combination of valvular endothelial cells and collagen matrix is combined in a $3 \mathrm{D}$ configuration.

\section{Conflict of Interests}

The authors declared no potential conflict of interests with respect to the authorship and publication of this paper.

\section{Acknowledgments}

Funding for this project was provided by Dr. Gopal Pande from the Council of Scientific and Industrial Research (Grant Code ESC 103) and by Dr. Anasuya Ganguly from Aditya Birla Science and Technology Group.

\section{References}

[1] M. Misfeld and H.-H. Sievers, "Heart valve macro- and microstructure," Philosophical Transactions of the Royal Society B: Biological Sciences, vol. 362, no. 1484, pp. 1421-1436, 2007.

[2] M. D. Combs and K. E. Yutzey, "Heart valve development: regulatory networks in development and disease," Circulation Research, vol. 105, no. 5, pp. 408-421, 2009.

[3] J. Lincoln, A. W. Lange, and K. E. Yutzey, "Hearts and bones: shared regulatory mechanisms in heart valve, cartilage, tendon, and bone development," Developmental Biology, vol. 294, no. 2, pp. 292-302, 2006.

[4] J. Lincoln, C. M. Alfieri, and K. E. Yutzey, "Development of heart valve leaflets and supporting apparatus in chicken and mouse embryos," Developmental Dynamics, vol. 230, no. 2, pp. 239250, 2004.

[5] H. Tseng and K. J. Grande-Allen, "Elastic fibers in the aortic valve spongiosa: a fresh perspective on its structure and role in overall tissue function," Acta Biomaterialia, vol. 7, no. 5, pp. 2101-2108, 2011.

[6] S. F. Badylak, D. Taylor, and K. Uygun, "Whole-organ tissue engineering: decellularization and recellularization of threedimensional matrix scaffolds," Annual Review of Biomedical Engineering, vol. 13, pp. 27-53, 2011.

[7] A. G. Kidane, G. Burriesci, P. Cornejo et al., "Current developments and future prospects for heart valve replacement therapy," Journal of Biomedical Materials Research Part B Applied Biomaterials, vol. 88, no. 1, pp. 290-303, 2009.

[8] K. Mendelson and F. J. Schoen, "Heart valve tissue engineering: concepts, approaches, progress, and challenges," Annals of Biomedical Engineering, vol. 34, no. 12, pp. 1799-1819, 2006.

[9] C. Pisano, E. Maresi, C. R. Balistreri et al., "Histological and genetic studies in patients with bicuspid aortic valve and ascending aorta complications," Interactive Cardiovascular and Thoracic Surgery, vol. 14, no. 3, pp. 300-306, 2012.

[10] B. Weber, M. Y. Emmert, and S. P. Hoerstrup, "Stem cells for heart valve regeneration," Swiss Medical Weekly, vol. 142, Article ID w13622, 2012.
[11] B. Weber, M. Y. Emmert, R. Schoenauer, C. Brokopp, L. Baumgartner, and S. P. Hoerstrup, "Tissue engineering on matrix: future of autologous tissue replacement," Seminars in Immunopathology, vol. 33, no. 3, pp. 307-315, 2011.

[12] P. Zilla, J. Brink, P. Human, and D. Bezuidenhout, "Prosthetic heart valves: catering for the few," Biomaterials, vol. 29, no. 4, pp. 385-406, 2008.

[13] W.-H. Zimmermann and T. Eschenhagen, "Tissue engineering of aortic heart valves," Cardiovascular Research, vol. 60, no. 3, pp. 460-462, 2003.

[14] L. G. Luna, Manual of Histologic Staining Methods of the Armed Forces Institute of Pathology, McGraw-Hill, 3rd edition, 1968.

[15] V. Nanduri, S. M. Tattikota, R. T. Avinash, V. R. Sriramagiri, S. Kantipudi, and G. Pande, "Reconstruction of hyaline cartilage deep layer properties in 3-dimensional cultures of human articular chondrocytes," Orthopaedic Journal of Sports Medicine, vol. 2, no. 6, 2014.

[16] S. Thirion and F. Berenbaum, "Culture and phenotyping of chondrocytes in primary culture," Methods in molecular medicine, vol. 100, pp. 1-14, 2004.

[17] E. M. Glare, M. Divjak, M. J. Bailey, and E. H. Walters, “ $\beta$ actin and GAPDH housekeeping gene expression in asthmatic airways is variable and not suitable for normalising mRNA levels," Thorax, vol. 57, no. 9, pp. 765-770, 2002.

[18] H. Hatano, A. Maruo, M. E. Bolander, and G. Sarkar, "Statin stimulates bone morphogenetic protein-2, aggrecan, and type 2 collagen gene expression and proteoglycan synthesis in rat chondrocytes," Journal of Orthopaedic Science, vol. 8, no. 6, pp. 842-848, 2003.

[19] H. Sekiguchi, N. Hemmi, T. Maki et al., "Culture on a fragmin/protamine-coated plate suppresses the collagen type I $\alpha$ I and TGF- $\beta 1$ mRNA expression of rat hepatic stellate RI-T cells," Journal of Veterinary Medical Science, vol. 75, no. 5, pp. 553-559, 2013.

[20] K. B. Kim, C. S. Hwang, C. G. Kim, and H. H. Jung, "Quantitative analysis of VCAM-1 mRNA expression levels in nasal mucosa of TDI-induced allergic rats," Journal of Rhinology, vol. 5, no. 2, pp. 134-137, 1998.

[21] N. H. Machell, O. W. Blaschuk, and R. Farookhi, "Developmental expression and distribution of $\mathrm{N}$ - and $\mathrm{E}$-cadherin in the rat ovary," Biology of Reproduction, vol. 63, no. 3, pp. 797-804, 2000.

[22] V. Decot, G. Woerly, M. Loyens et al., "Heterogeneity of expression of IgA receptors by human, mouse, and rat eosinophils," Journal of Immunology, vol. 174, no. 2, pp. 628-635, 2005.

[23] A. C. Liu, V. R. Joag, and A. I. Gotlieb, "The emerging role of valve interstitial cell phenotypes in regulating heart valve pathobiology," The American Journal of Pathology, vol. 171, no. 5, pp. 1407-1418, 2007.

[24] M. S. Sacks, F. J. Schoen, and J. E. Mayer Jr., "Bioengineering challenges for heart valve tissue engineering," Annual Review of Biomedical Engineering, vol. 11, pp. 289-313, 2009.

[25] F. J. Schoen, "Evolving concepts of cardiac valve dynamics: the continuum of development, functional structure, pathobiology, and tissue engineering," Circulation, vol. 118, no. 18, pp. 18641880, 2008.

[26] C. Y. Y. Yip and C. A. Simmons, "The aortic valve microenvironment and its role in calcific aortic valve disease," Cardiovascular Pathology, vol. 20, no. 3, pp. 177-182, 2011. 

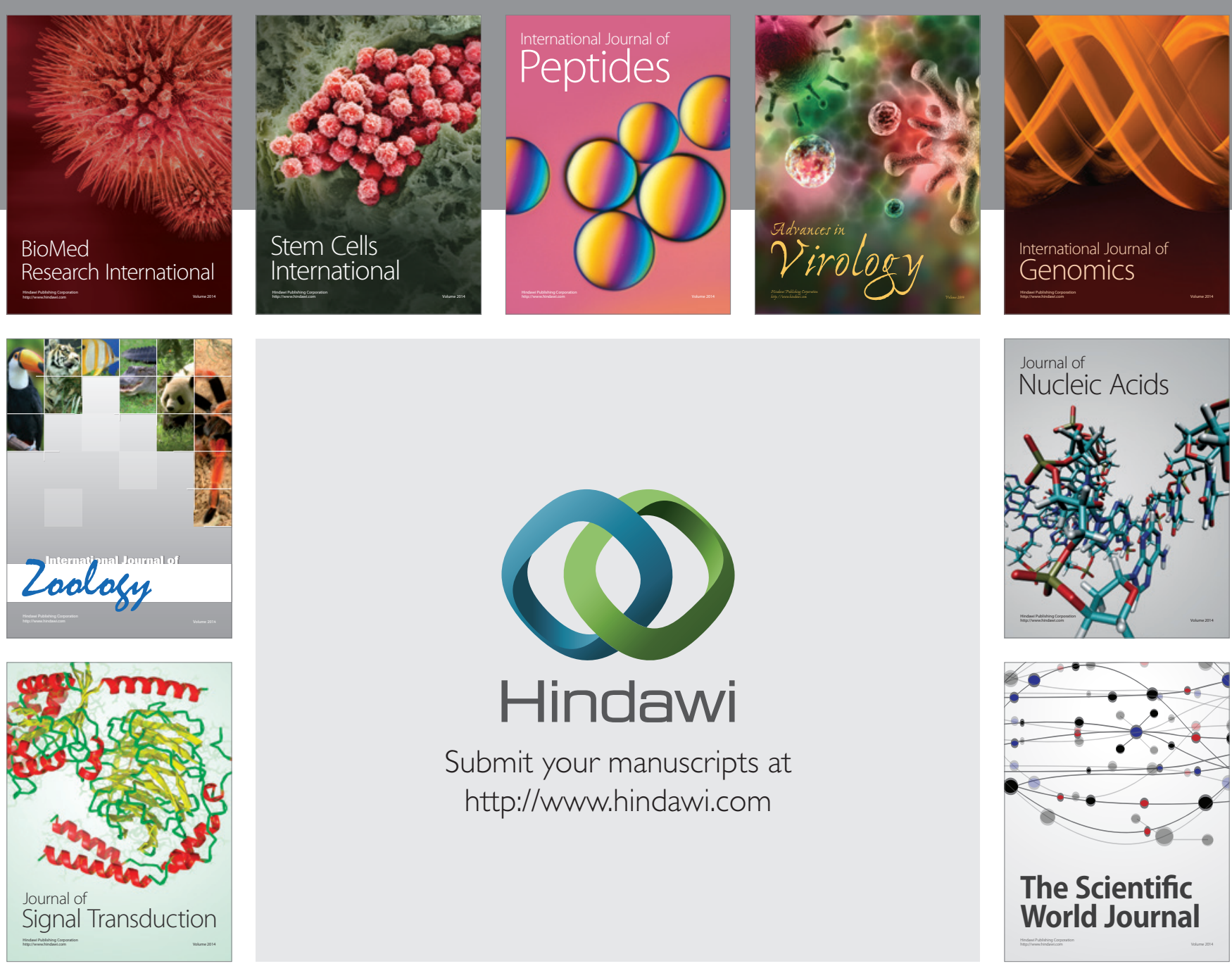

Submit your manuscripts at

http://www.hindawi.com
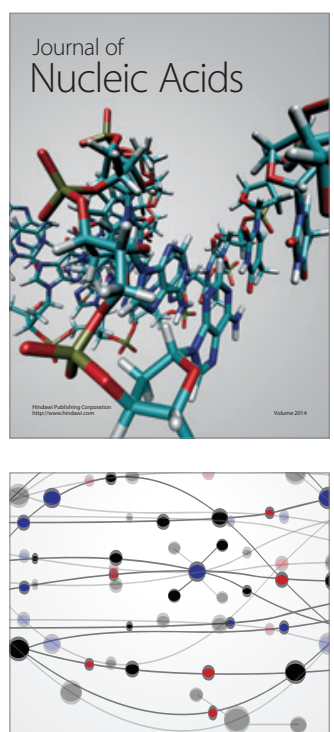

The Scientific World Journal
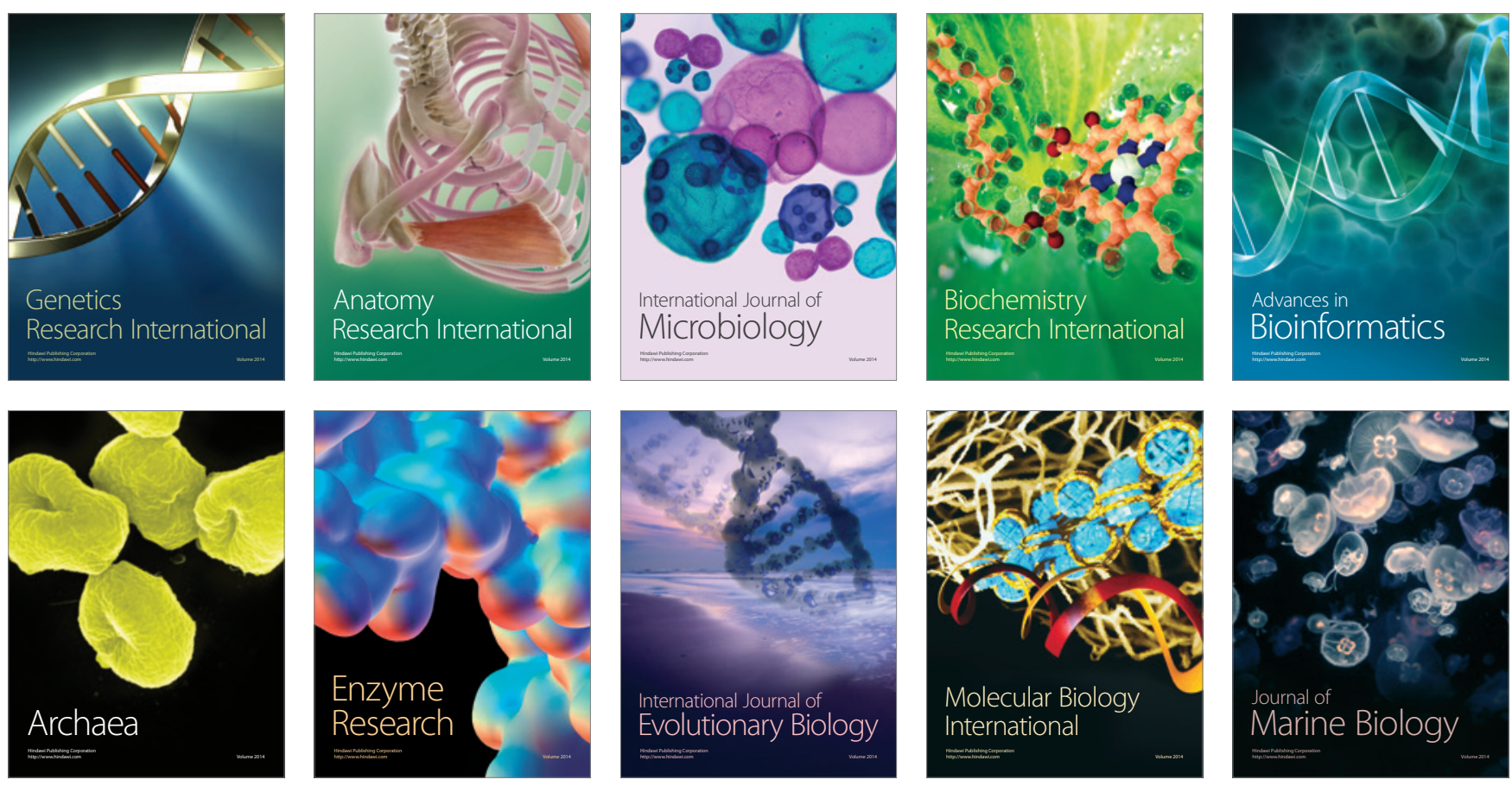